\title{
La productivité naturelle et la réglementation comme déterminants de la valeur du foncier ostréicole : Un modèle de prix hédoniques spatial appliqué au Bassin d'Arcachon
}

\author{
Gabrielle Lesur-Irichabeau ${ }^{1,2}$ \\ ${ }^{1}$ Post-doctorante, UMR AMURE - IFREMER Centre de Brest, Département d'Economie Maritime, PLOUZANE \\ ${ }^{2}$ Chercheur affilié au GREThA UMR CNRS 5113, Université Montesquieu Bordeaux IV, Avenue Léon Duguit, \\ PESSAC \\ *: Corresponding author : Gabrielle Lesur-Irichabeau, email address : Gabrielle.Lesur.Irichabeau@ifremer.fr
}

\section{Résumé :}

Afin de montrer l'importance de la réglementation et de la productivité naturelle dans la valorisation des surfaces de production ostréicoles, nous avons estimé une fonction de prix hédonique des concessions ostréicoles arcachonnaises. Les « terres ostréicoles » ont comme particularité d'être des concessions du Domaine Public Maritime et sont à ce titre soumises à son régime protecteur. Néanmoins, loin d'entraver l'activité ostréicole par les contraintes qu'elle impose, la réglementation se révèle être une garante du maintien d'un niveau satisfaisant de productivité naturelle des concessions pour permettre une reproduction et une croissance des huîtres dans des conditions favorables. Ainsi, les résultats de la modélisation montrent que plus de la moitié du prix d'échange des concessions est déterminé par les conditions réglementaires régissant leur utilisation. Ils soulignent également que les conditions du milieu ne sont pas identiques sur l'ensemble du Bassin d'Arcachon justifiant une réglementation adaptée aux différences de productivité naturelle.

\begin{abstract}
:
To demonstrate the importance of regulation and the natural productivity in the economic valuation of oyster production areas, we estimated a hedonic price function of oyster leases in Arcachon Bay. The "oyster land" have the particularity to be concessions of Maritime Public Domain and are therefore subject to its protective system. Nevertheless, far from hindering the oyster farming by the constraints it imposes, the regulation appears to be a guarantor of maintaining a level of natural productivity concessions allowing a satisfactory reproduction and growth of oysters in favorable conditions. Thus, the modeling results show that more than half the price of trade concessions is determined by the regulatory requirements governing their use. They also show that environmental conditions are not identical across the Bay of Arcachon, while justifying the regulation has to be adaptive to the natural productivity differences.
\end{abstract}




\section{Introduction}

Les surfaces de production ostréicoles sont un facteur de production pour l'ostréiculture au même titre que la terre pour l'agriculture. Néanmoins, elles ont comme particularité d'être des concessions du Domaine Public Maritime (DPM) et sont à ce titre soumises aux critères de la domanialité publique et aux contraintes de son régime protecteur. Tout comme les autres biens appartenant au Domaine Public, les "terres ostréicoles " sont soumises aux principes d'imprescriptibilité et d'inaliénabilité. Un droit spécial peut cependant être accordé sous des conditions strictes d'attribution et de durée. Ces droits spéciaux prennent la forme de concessions du Domaine Public. Normalement, en raison de leur statut de bien public, ces concessions ne peuvent pas faire l'objet de transactions entre personnes privées disposant d'un droit d'usage légal parce que l'existence de telles transactions reviendrait à reconnaître non plus un droit d'usage mais un droit de propriété. Néanmoins, pour les concessions ostréicoles, les transactions ont été autorisées par la loi en permettant à un concessionnaire de transférer son droit d'usage à d'autres concessionnaires, transferts accompagnés d'une contrepartie financière ${ }^{1}$, afin de formaliser et d'encadrer une pratique existante (Mongruel, et al., 2008a, 2008b). Ainsi, il existe un marché des concessions ostréicoles constitué des transferts du droit d'usage donnant lieu au versement d'une indemnité ${ }^{2}$.

Les surfaces de production ostréicoles se caractérisent donc par le fait qu'elles ont une valeur marchande mais aussi par le fait qu'elles sont situées sur un espace dont l'usage n'est pas exclusif - justifiant ainsi en partie leur appartenance au Domaine Public - comme c'est le cas du Bassin d'Arcachon qui est connu non seulement pour son ostréiculture mais également pour son tourisme et ses activités nautiques et de pêche (professionnelle ou non). Ces activités concurrentes peuvent être à l'origine de conflits autour de l'usage de l'eau mais aussi de sa qualité. En effet, la qualité de l'eau est une condition essentielle à la production ostréicole à la fois en raison de l'existence d'une classification sanitaire des zones de production qui détermine les conditions de commercialisation des huîtres, mais aussi en raison du fait que selon la qualité de l'eau, la productivité naturelle du milieu va être plus ou moins favorable à la croissance et à la survie des huîtres reflétant ainsi la dépendance de l'ostréiculture au milieu naturel. Comme cela a pu être le cas dans le passé avec la crise ostréicole du milieu des années 1970 liée à la pollution de l'eau par les peintures anti-salissures des bateaux, les activités concurrentes peuvent donc être une source d'externalités négatives.

Nous nous intéressons ici à la valeur marchande des surfaces de production ostréicoles en tant que reflet de la qualité du milieu ainsi que de sa productivité. Afin d'intégrer ces aspects environnementaux non quantifiables, l'étude de la valeur du foncier nécessite de mobiliser des méthodes d'évaluation non marchande des biens. Le recours à l'évaluation par la méthode des prix hédoniques permet alors de différencier les déterminants réglementaires et ceux liés à la productivité naturelle

\footnotetext{
${ }^{1}$ Cette compensation financière est appelée « indemnité de substitution » ou « indemnité de cession $»$.

${ }^{2}$ Le marché n'est pas le seul canal d'acquisition de concessions ostréicoles. En effet, il existe des transmissions et des échanges (dans le cadre familial notamment mais pas exclusivement) sans contrepartie financière ou à un prix inférieur à celui du marché ainsi que des attributions gratuites par l'Etat, principalement à destination des nouvelles installations. Notre étude portant sur les indemnités de cession, nous nous intéressons donc qu'au seul marché des concessions. Le lecteur intéressé par le fonctionnement des transmissions pourra se reporter aux analyses de Dumont (1986) et Mongruel, et al. (2008a, 2008b).
} 
des concessions du prix de ces dernières ${ }^{3}$. Elle permet donc de révéler les composantes hors marché d'un prix. Si ces méthodes sont couramment utilisées dans les analyses foncières et immobilières relatives à l'habitat, elles n'ont jamais été utilisées dans le cas des concessions ostréicoles et cela principalement parce que les études économiques de l'activité ostréicole sont relativement rares. En effet, à notre connaissance, seuls Dumont (1986) puis Mongruel, et al., (2008a; 2008b) ont abordé cette question, mais uniquement d'un point de vue théorique.

La méthode des prix hédonistes, initiée par Ridker et Henning (1967), repose sur l'idée que la valeur attribuée par les individus à la qualité de l'environnement peut être estimée à partir des sommes monétaires qu'ils consacrent à l'achat de biens intégrants des caractéristiques d'environnement. Cette méthode, en se fondant sur la considération que le prix d'un bien - ici une concession ostréicole - dépend des caractéristiques du bien en tant que facteur de production (valeur intrinsèque) mais aussi des avantages que procure le milieu dans lequel il se trouve, permet de révéler la valeur implicite accordée à l'environnement.

Généralement, la méthode des prix hédonistes est appliquée à des biens de consommation différenciés ${ }^{4}$ tels que les habitations (Rosen, 1974$)^{5}$ mais on trouve également des applications pour les facteurs de production différenciés que sont les terres agricoles (Palmquist, 1989). Avant Palmquist (1989) des travaux empiriques ${ }^{6}$ avaient déjà été menés sur cette question de l'évaluation du prix des facteurs de production différenciés par la méthode des prix hédoniques mais pas de façon détaillée. Palmquist (1989) part du fait que l'on considère généralement les terres comme un facteur de production homogène alors que chaque terre a des caractéristiques qui peuvent varier selon les secteurs. Cela inclut des caractéristiques pouvant être modifiées (l'irrigation, les aménagements des terres, etc.) et des caractéristiques qui ne peuvent pas être modifiées (la structure du sol par exemple). Le prix de la terre dépendrait alors de ces différentes caractéristiques.

Aussi, la production primaire d'un milieu marin n'est pas extensive; une surexploitation des ressources trophiques par une surcharge en animaux en élevage peut créer des externalités négatives entre concessionnaires en réduisant la disponibilité en ressources alimentaires pour les huîtres de chaque concession. Ces externalités, connues également sous le terme d'externalités de congestion, font références à la Tragédie des Communs (Hardin, 1968). En effet, les concessionnaires qui partagent un même niveau de ressources trophiques disponibles vont voir leur utilité décroître à mesure que va s'accroître la biomasse d'huîtres en élevage. Tous ces éléments renvoient ainsi à la question de la rente différentielle extensive telle que définie par Ricardo. La rente est alors le prix du droit d'usage d'une terre et de ses forces productives et cette rente est différentielle dans le sens où la terre peut différer en force productive (Ricardo, 1819). Le prix de la terre reflète donc les différences de productivité de cette dernière.

L'existence de différences de productivité et d'externalités de congestion a justifié que des mesures de restriction de densité aient été imposées afin de limiter les

\footnotetext{
${ }^{3}$ L'ensemble des hypothèses sous-jacentes à l'utilisation d'un tel modèle et notamment celle relative à un marché en situation de concurrence pure et parfaite ou encore celle relative à l'existence d'un continuum de valeurs possibles pour les différentes caractéristiques des biens sont considérées comme satisfaites.

${ }^{4}$ Un bien différencié est défini par l'ensemble de ses caractéristiques constitutives (Le Goffe, 1996).

${ }^{5}$ Un modèle théorique alternatif a été développé également par Freeman en 1974 mais si les deux sont utilisés, celui de Rosen est le plus cité (Palmquist, 1989).

${ }^{6}$ Il s'agit notamment des travaux de Downing (1973) ou encore de Chicoine (1981).
} 
risques de surcharge des concessions ostréicoles. Ces restrictions, prises par l'administration en charge des Affaires Maritimes, sont régionalisées (chaque bassin de production a ses propres règles d'usage des concessions et notamment en termes de densité) mais sont aussi localement différenciées afin de tenir compte des différences de productivité liées à la localisation géographique des concessions.

\section{La réglementation locale : les schémas des structures}

L'établissement d'un schéma des structures destiné entre autres à prendre toutes les dispositions qui seront nécessaires afin de "favoriser une meilleure répartition des eaux salées nécessaires aux productions biologiques " a été imposé par le décret du 14 septembre 1987 qui vient modifier celui du 22 mars 1983 ${ }^{7}$. Les schémas des structures ${ }^{8}$ imposent notamment des restrictions de densité pour l'élevage et le captage qui peuvent par ailleurs être différentes selon le secteur concerné. La Carte $1^{9}$ suivante distingue au sein du cadastre ostréicole arcachonnais les différents secteurs soumis à des dispositions particulières (Courbey, Grand Banc, Bélisaire et Mimbeau).

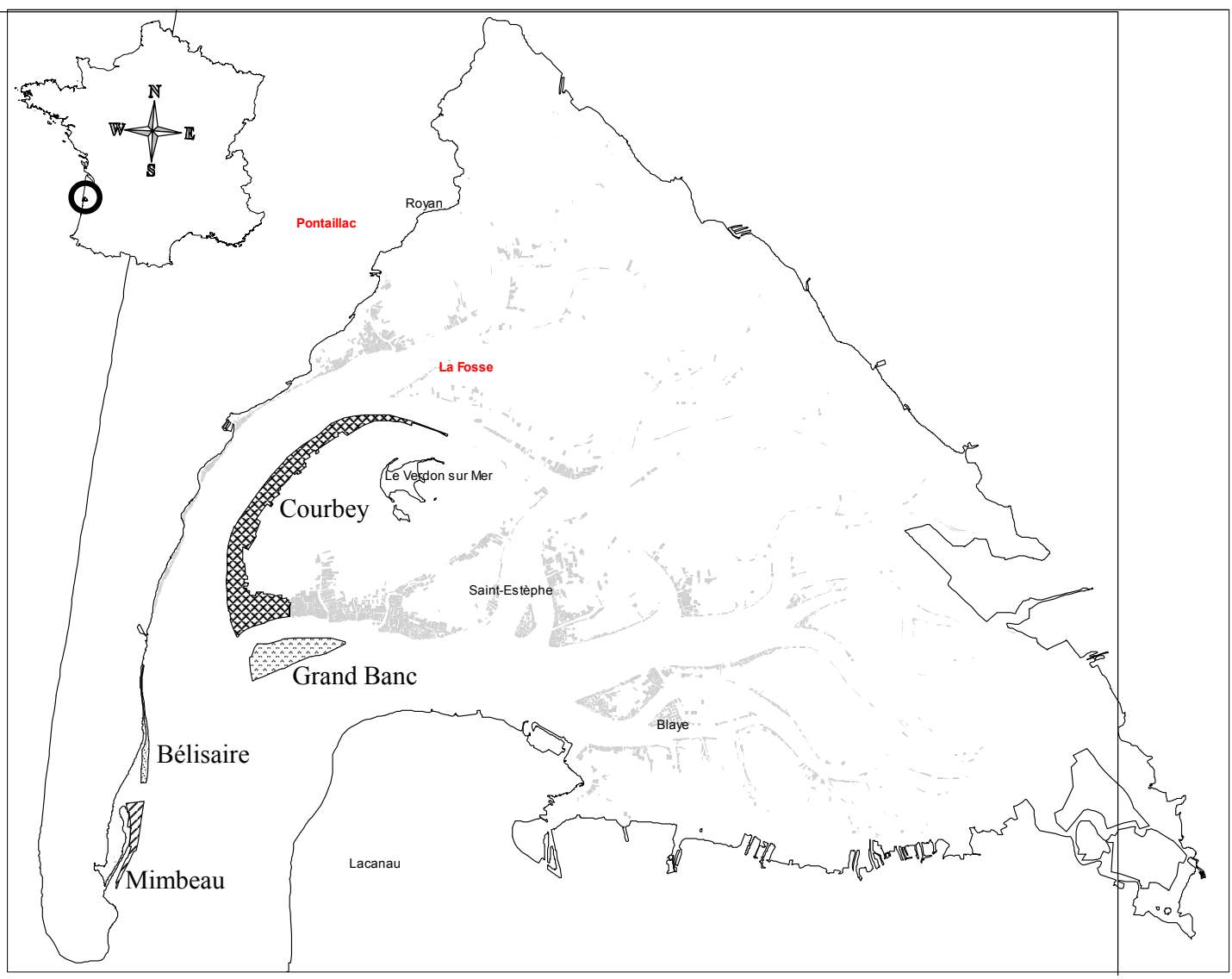

\footnotetext{
${ }^{7}$ Décret du 22 mars 1983 « fixant le régime de l'autorisation des exploitations de cultures marines $"$.

${ }^{8}$ Le lecteur intéressé par une description plus complète des différents schémas des structures du Bassin d'Arcachon pourra se reporter aux travaux de Irichabeau (2011).

${ }^{9}$ Toutes les cartes ont été réalisées par l'auteur sous Mapinfo à partir de données mises à la disposition par les Affaires Maritimes de la Gironde et/ou l'IFREMER et/ou construites par l'auteur.
} 
Carte 1. Secteurs soumis à des dispositions particulières au titre des schémas des structures

Les principales dispositions particulières instituées à partir de 1992 sont les suivantes :

- Bélisaire : densité maximale d'une tonne à l'are et $50 \%$ de la surface au maximum est occupée par les poches.

- Grand Banc : densité maximale d'une tonne à l'are pour l'élevage à plat et $50 \%$ de la surface au maximum est occupée par les poches en surélevé. Le captage sur tables ne doit pas dépasser également $50 \%$ de la surface concédée.

- Mimbeau : densité maximale d'une tonne à l'are pour l'élevage à plat et $50 \%$ de la surface au maximum est occupée par les poches en surélevé.

- Courbey : densité maximale de $800 \mathrm{~kg}$ à l'are pour l'élevage à plat et $50 \%$ de la surface au maximum est occupée par les poches en surélevé.

Pour les secteurs non soumis à des dispositions particulières, à partir de 1992, le captage ne pourra être effectué que sur $25 \%$ de la surface concédée à un exploitant. Cette surface passera en 2009 à $40 \%$ avec de plus une restriction sur la quantité de collecteur pouvant être posée. Ainsi, le nombre de collecteur est limité à 1000 équivalent-tuiles ${ }^{10}$. Quant aux restrictions de densité pour l'élevage, elles sont instituées à hauteur de 100 poches à l'are pour l'élevage en surélevé et 1 tonne à l'are pour l'élevage à plat.

L'introduction de dispositions particulières dans les schémas des structures tendent à limiter davantage l'activité sur les parcelles sur lesquelles elles s'appliquent. Elles s'appliquent donc aux secteurs où le risque d'un déséquilibre dans la répartition des eaux est le plus important, les secteurs concernés ayant été identifiés comme les plus productifs. En effet, comme le constataient Hérald, et al. (1986) avant même la mise en place des schémas des structures, il est nécessaire de limiter la biomasse en élevage afin de préserver voire d'améliorer les niveaux de productivité. II existe par conséquent un lien entre la réglementation (au travers des schémas des structures) et la productivité du milieu, la première ayant pour but d'optimiser la seconde. Le prix d'une parcelle va donc à la fois refléter la réglementation qui s'applique à cette dernière et l'environnement naturel dont elle bénéficie.

Les écarts de productivité (et donc de prix) et le lien entre le prix et la réglementation qui s'applique en matière d'exploitation sont parfaitement illustrés par la Carte 2 suivante. Les secteurs les plus valorisés correspondent en effet à ceux soumis à des dispositions particulières.

\footnotetext{
${ }^{10}$ Plusieurs types de collecteurs peuvent être utilisés : des tuiles, des coupelles, des tubes, etc. Pour simplifier, nous exprimons le nombre de collecteurs en équivalent-tuile.
} 


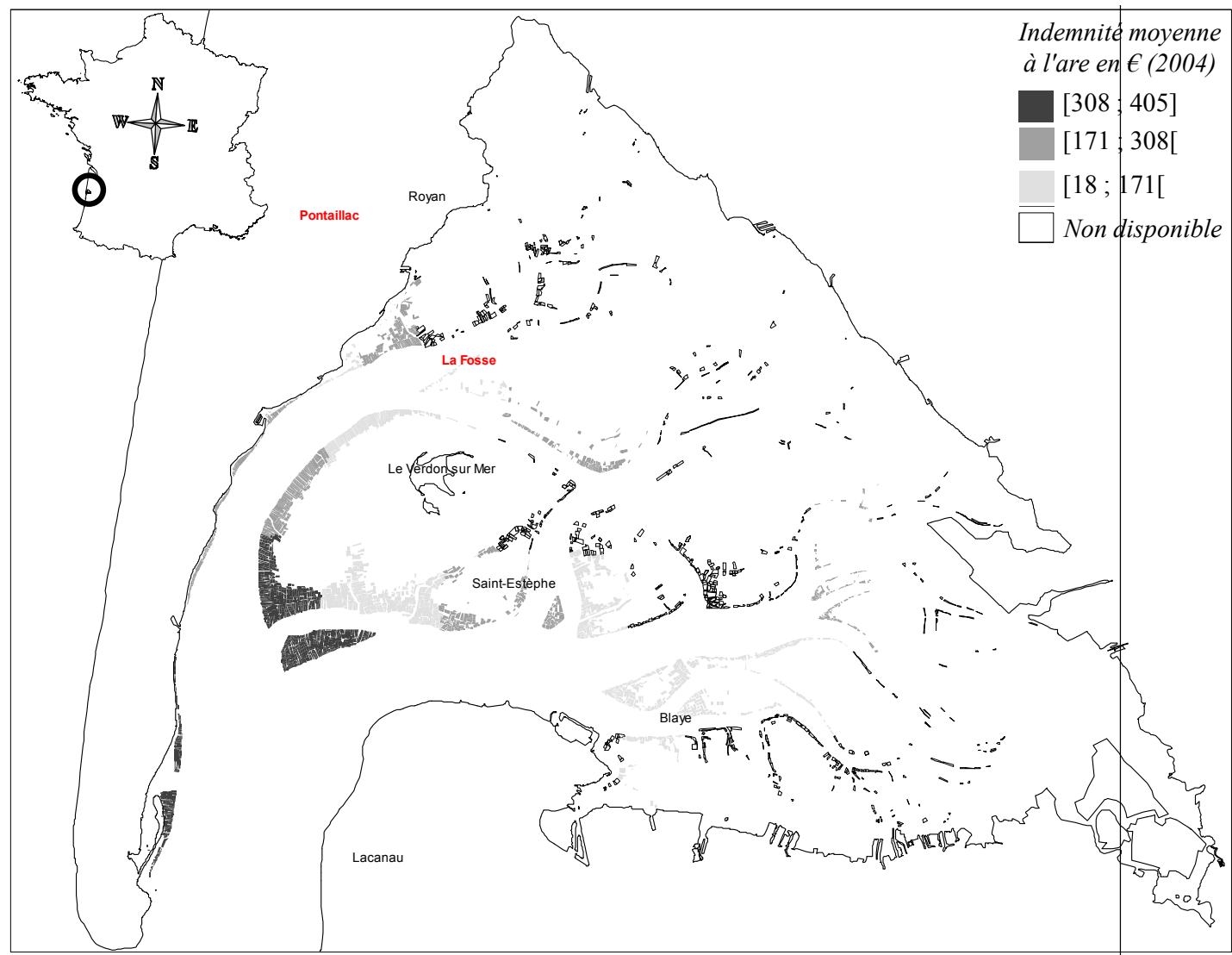

Carte 2. Répartition géographique des indemnités de substitution à l'are en 2004 dans le Bassin d'Arcachon ${ }^{11}$.

Cette carte nous laisse supposer par ailleurs l'existence d'une concentration spatiale des prix ; la distribution des indemnités de substitution n'est pas aléatoirement réalisée dans l'espace du Bassin d'Arcachon, les parcelles de même valeur se situant à proximité les unes des autres (les valeurs les plus élevées étant relevées au sud du Bassin, les moins élevées concernant des parcelles plus à l'intérieur du Bassin). II est donc nécessaire de mobiliser des outils statistiques appropriés afin de détecter la présence d'une autocorrélation spatiale. Si cette présence est confirmée, le modèle de prix hédoniques des concessions ostréicoles devra être adapté.

\section{Analyse exploratoire de données spatiales}

Les méthodes d'analyse exploratoire de données spatiales - ou ESDA pour Exploratory Spatial Data Analysis - permettent d'identifier l'existence d'un lien géographique entre les observations appelé autocorrélation spatiale. L'autocorrélation spatiale est une mesure de la dépendance entre les valeurs d'une variable aléatoire mesurées sur des unités spatiales, dépendance qui s'explique par leur proximité.

Les méthodes généralement utilisées pour la détection de l'autocorrélation spatiale sont le diagramme de Moran (Anselin, 1996) et les indicateurs locaux d'association

\footnotetext{
${ }^{11}$ Nous disposons aussi d'informations sur les indemnités moyennes en 2010 mais pas sur toutes les zones du Bassin c'est pourquoi nous avons préféré ne faire figurer que les informations relatives à l'année 2004.
} 
spatiale ou LISA ${ }^{12}$ (Anselin, 1995b). La première permet de détecter l'autocorrélation spatiale globale tandis que la seconde détecte l'autocorrélation spatiale locale $e^{13} .410$ transactions sont étudiées (Carte 3). Ces transactions ont eu lieu entre 1988 et $2010^{14}$. L'indemnité la plus faible est de $69 €$ tandis que l'indemnité la plus élevée est de 41 108€. L'indemnité moyenne est de 5 604€.

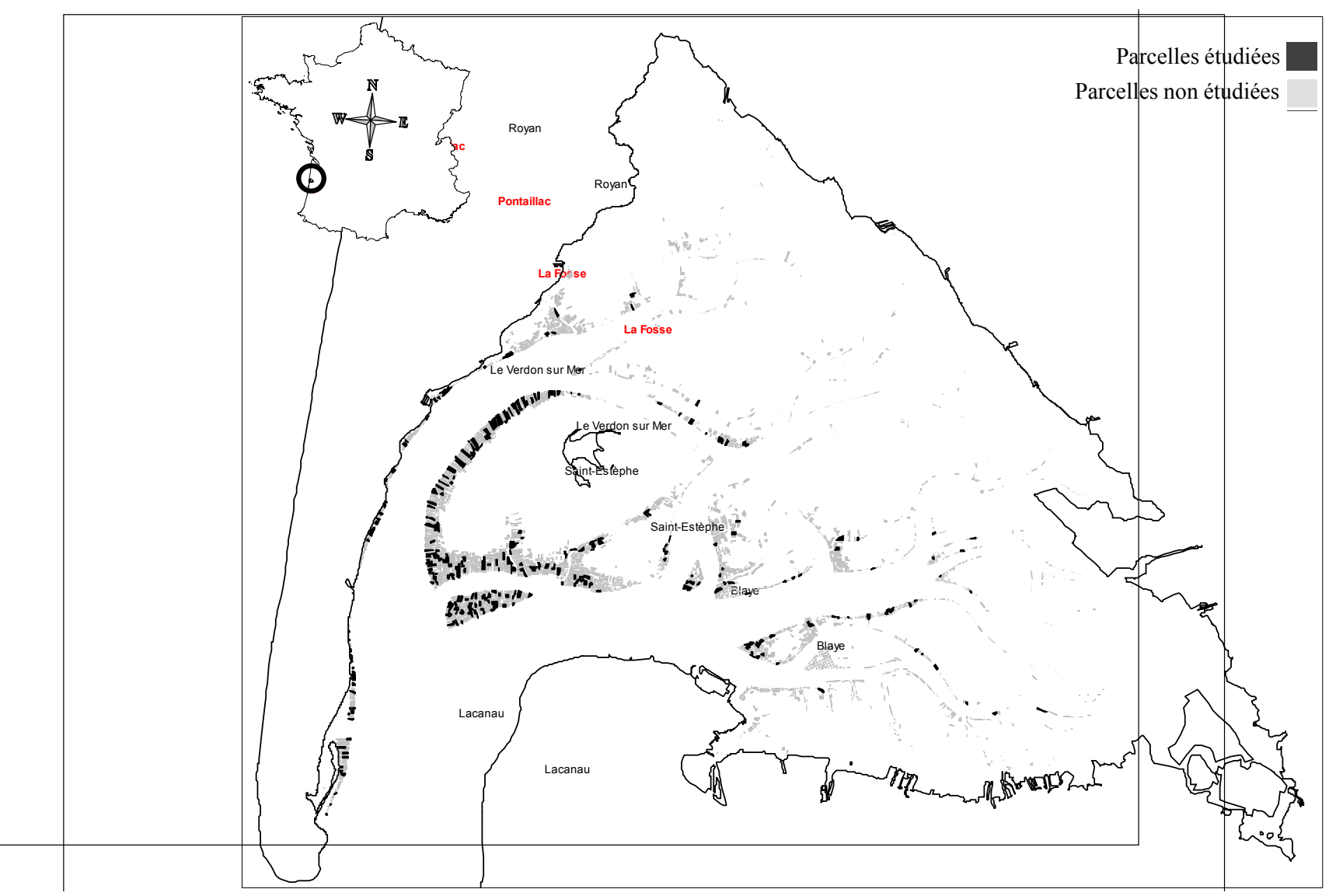

Carte 3. Parcelles du cadastre ostréicole arcachonnais étudiées

Afin de calculer le I de Moran ${ }^{15}$ (Moran, 1948; 1950) qui représente l'autocorrélation spatiale et d'appliquer les méthodes de l'analyse exploratoire de données spatiales, nous avons choisi d'utiliser une matrice de poids ${ }^{16}$ des 8 plus proches voisins ${ }^{17}$.

\footnotetext{
${ }^{12}$ Local Indicators of Spatial Association.

${ }^{13}$ Le logiciel utilisé pour la mise en œuvre de l'analyse exploratoire de données spatiales est OpenGeoDA téléchargeable à l'adresse suivante : http://geodacenter.asu.edu/. Pour des informations relatives à son utilisation, voir Anselin (1995a).

${ }^{14}$ En raison du nombre d'années considéré, les montants des indemnités de substitution ont été actualisés et sont exprimés en euros 2010. Le foncier ostréicole ne pouvant être assimilé à un marché foncier ou immobilier classique, l'actualisation des prix a été réalisée à partir de l'évolution des prix à la consommation.

${ }^{15}$ La formulation du / de Moran est donnée en Annexe.

${ }^{16}$ Une présentation sommaire des matrices de poids est donnée en Annexe.

${ }^{17}$ Nous avons testé plusieurs matrices de poids : une matrice de distance euclidienne et différentes matrices des k-plus proches voisins. La présence d'unités spatiales isolées ne nous permettait pas d'utiliser une matrice de contiguïté. Le choix de la matrice de poids a été réalisé en retenant celle dont, compte-tenu de la structure spatiale des données, la valeur du I de Moran standardisée associée est la plus élevée (Dall'Erba, 2004). Le tableau A porté en Annexe présente les statistiques / de Moran pour les différentes matrices de poids testées.
} 
Cette matrice de poids représente la façon dont deux localisations sont connectées spatialement.

Les résultats de l'étude de l'autocorrélation globale sont alors donnés par le diagramme de Moran ${ }^{18}$ suivant (Figure 1).

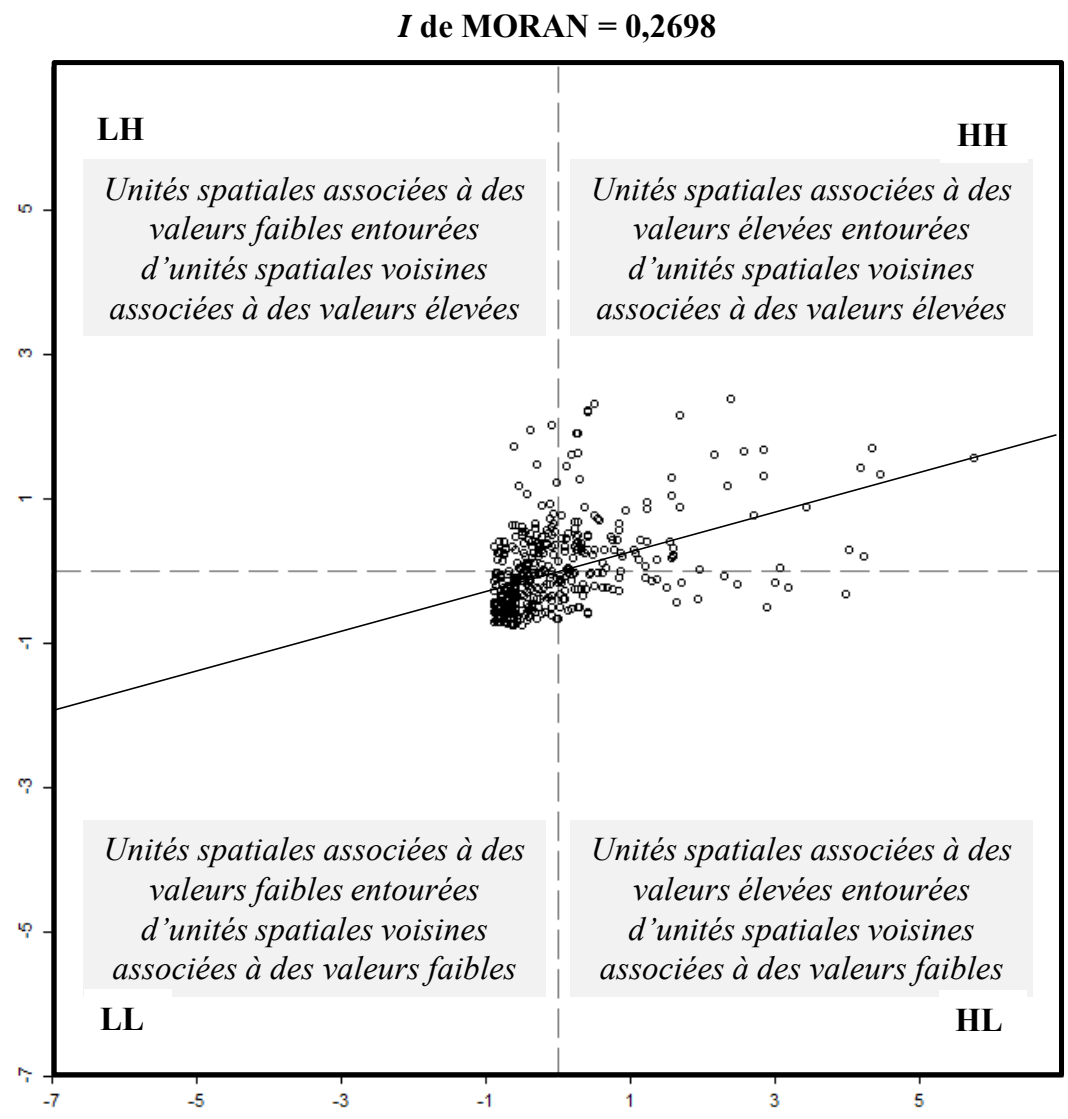

Figure 1. Résultats de l'étude de l'autocorrélation spatiale globale

Ainsi, ce diagramme nous indique que l'on est en présence d'autocorrélation spatiale ; cette dernière est positive (pour $67 \%$ des concessions étudiées) ce qui signifie que globalement, les parcelles associées à des indemnités élevées (respectivement faibles) sont à proximité de parcelles associées également à des indemnités élevées (respectivement faibles).

Les parcelles appartenant au quadrant $\mathrm{HH}$ représentent $22 \%$ des parcelles étudiées avec une indemnité moyenne de $12580 €$. $92 \%$ d'entre elles sont soumises à des dispositions particulières et sont donc situées principalement dans le sud du Bassin. Celles appartenant au quadrant LL représentent $45 \%$ des concessions étudiées avec une indemnité moyenne de $1993 €$; elles ne sont que $26 \%$ à être soumises à des dispositions particulières et sont localisées principalement dans la partie

\footnotetext{
${ }^{18}$ Dans le diagramme de Moran figure en abscisse la valeur standardisée de la variable étudiée notée $W$ et son décalage spatial standardisé $W y$. Les points qui représentent les observations sont alors répartis selon le signe de leurs coordonnées. Le diagramme de Moran s'interprète en analysant la répartition des points dans les différents quadrants. La pente de la droite représente l'indice de Moran.
} 
intérieure du Bassin, loin des passes ${ }^{19}$. Les localisations atypiques du quadrant LH représentent $20 \%$ des parcelles étudiées, avec une indemnité moyenne de $3143 €$; elles sont situées plutôt à proximité des passes expliquant que $76 \%$ d'entre elles sont soumises à des dispositions particulières. Enfin, les concessions du quadrant $\mathrm{HL}$ représentent $13 \%$ des parcelles étudiées avec une indemnité moyenne de $10258 €$. Un peu plus de la moitié sont concernées par des dispositions particulières. Elles sont situées principalement au Nord et Nord-Ouest du Grand Banc, le long du principal chenal du Bassin, et sont moins concentrées spatialement que les parcelles des autres quadrants expliquant ainsi la présence d'indemnité de faible niveau dans le quadrant $\mathrm{HL}$. A nouveau, cela nous indique que les parcelles les plus valorisées sont situées à proximité des passes du Bassin d'Arcachon.

Cette analyse globale doit être complétée par une analyse locale afin de détecter, au sein du schéma global, des concentrations géographiques significatives de valeurs similaires et si certaines observations et leurs voisines se détachent de cette tendance, ce que permettent de faire les indicateurs locaux d'association spatiale ${ }^{20}$.

Les résultats de cette analyse locale de l'autocorrélation, suite aux 9999 permutations réalisées, indiquent qu'un peu plus d'un tiers (34\%) des parcelles ont une association spatiale significative. $10 \%$ sont significatives au seuil de $0,01 \%, 21 \%$ au seuil de $0,10 \%, 29 \%$ au seuil de $1 \%$ et $40 \%$ au seuil de $5 \%$. L'analyse des clusters quant à elle met en évidence le fait que les parcelles associées à un LISA significatif relèvent d'un schéma local d'association spatiale reflétant une autocorrélation spatiale positive puisque plus de $84 \%$ de ces parcelles appartiennent aux quadrants $\mathrm{HH}$ ou LL avec toutefois près de $60 \%$ de ces dernières dans le quadrant LL. La totalité des parcelles associées à un LISA significatif du quadrant $\mathrm{HH}$ sont soumises à dispositions particulières. Celles associées à un LISA significatif du quadrant LL sont quant à elles moins de $9 \%$ à être concernées par des dispositions particulières. On a donc bien une cohérence entre les niveaux des indemnités et la soumission ou non à des dispositions particulières. Les dispositions particulières s'appliquent aux parcelles les plus productives qui par conséquent sont celles présentant une indemnité élevée.

Concernant les parcelles qui dévient du schéma global, toutes sont soumises à des dispositions particulières pour celles se trouvant dans le quadrant $\mathrm{LH}$; la présence de parcelles à indemnité faible au sein d'une zone où les parcelles ont des indemnités élevées s'explique par le fait que les surfaces concernées présentent des caractéristiques différences de celles que l'on peut généralement observer. Ainsi, les parcelles du quadrant LH servent à l'élevage à plat (70\%) ou sont destinées au captage. De plus, moins de $40 \%$ d'entre elles sont en 1 ère ligne. Enfin, une seule y est soumise parmi celles appartenant au quadrant $\mathrm{HL}$. Le fait pour une parcelle d'être soumise à des dispositions particulières semble donc influencer sur la valeur de cette dernière comme nous l'indiquait déjà la Carte 2.

La présence d'une autocorrélation spatiale dans la distribution des indemnités de substitution doit être intégrée dans la modélisation hédonique. Le modèle sera donc un modèle spatial de prix hédoniques des indemnités de substitution.

\footnotetext{
${ }^{19}$ Les « passes » du Bassin d'Arcachon correspondent à l'embouchure de ce dernier.

${ }^{20}$ La formulation des LISA est donnée en annexe.
} 


\section{Le modèle hédonique spatial des indemnités de substitution}

Etant donnée la présence d'une autocorrélation spatiale dans la distribution des indemnités de substitution, la modélisation économétrique va être réalisée grâce aux outils de l'économétrie spatiale ${ }^{21}$.

\subsection{Variables}

Les variables de notre modèle sont indiquées dans le Tableau 1 ; nous décrivons plus précisément ces dernières.

Nombre de transactions l'année précédant la date de la transaction (nbtn_1). Cette variable correspond au nombre total de transactions - y compris celles n'ayant pas donné lieu au versement d'une indemnité - réalisé l'année précédant la date de transaction de la parcelle concernée. On peut en effet penser qu'un nombre réduit de transactions l'année précédente entraînera une hausse du montant de l'indemnité de transaction l'année suivante et inversement.

21 Pour une présentation des différents modèles économétriques tenant compte de l'autocorrélation spatiale (SAR, SEM, SDM, SAC, etc.) et des problèmes spécifiques (autocorrélation, hétérogénéité, etc.) de l'économétrie spatiale, voir en particulier Anselin (1988; 1999), Fingleton \& Le Gallo (2008a ; 2008b), Le Gallo (2002 ; 2004), LeSage (1999). 


\begin{tabular}{|c|c|c|c|c|c|c|c|}
\hline Variable $^{a}$ & Intitulé & Unité & Minimum & Maximum & Moyenne & $\begin{array}{l}\text { Ecart- } \\
\text { type }\end{array}$ & Effet attendu ${ }^{b}$ \\
\hline INDEM $^{(1)}$ & Indemnité de substitution & $€_{2010}$ & 69 & 41108 & 5604 & 6170 & \\
\hline NBTN_1 $1^{(1,5)}$ & $\begin{array}{l}\text { Nombre de transactions en } \\
n-1\end{array}$ & Nombre & 18 & 109 & 68,20 & 26,20 & Négatif \\
\hline DISTKM $^{(5)}$ & $\begin{array}{l}\text { Distance au port le plus } \\
\text { proche }\end{array}$ & Kilomètre & 0,071 & 4,97 & 2,060 & 1,183 & Négatif \\
\hline $\mathrm{PCAPT}^{(1,3,5)}$ & Potentiel de captage & Equivalent-tuile & 440 & 126321 & 15739 & $\begin{array}{c}14 \\
056\end{array}$ & Positif \\
\hline $\mathrm{SAL}^{(2)}$ & Salinité & La salinité s'exprime sans unité & 27,49 & 33,81 & 32,01 & 1,15 & Négatif/Positif \\
\hline $\mathrm{MES}^{(2)}$ & Matières en suspension & $\mathrm{mg} / \mathrm{l}$ & 3,94 & 13,05 & 6,07 & 1,54 & Négatif/Positif \\
\hline $\mathrm{CHLA}^{(2)}$ & Chlorophylle a & $\mu g / l$ & 0,85 & 3,98 & 1,83 & 0,63 & Négatif/Positif \\
\hline $\mathrm{NH} 4^{(2)}$ & Ammonium & $\mu \mathrm{mol} / \mathrm{l}$ & 0,34 & 5,24 & 2,45 & 1,09 & Négatif/Positif \\
\hline $\mathrm{NIT}^{(2)}$ & Nitrates & $\mu \mathrm{mol} / \mathrm{l}$ & 0,06 & 20,54 & 5,38 & 3,31 & Négatif/Positif \\
\hline $\mathrm{TECH}^{(1,3)}$ & Technique d'élevage & 1 si à plat, 0 sinon & 0 & 1 & 0,26 & 0,44 & Négatif \\
\hline $\operatorname{CSANIT}^{(4)}$ & Classement sanitaire & 1 si classée $A, 0$ sinon & 0 & 1 & 0,90 & 0,30 & Positif \\
\hline ELEVDUM $^{(1,3)}$ & Utilisation de la parcelle & 1 si élevage, 0 sinon & 0 & 1 & 0,78 & 0,42 & Positif \\
\hline $\mathrm{ESPHC}(1,3)$ & Espèce présente & $\begin{array}{l}1 \text { si uniquement des huîtres } \\
\text { creuses, } 0 \text { sinon }\end{array}$ & 0 & 1 & 0,63 & 0,48 & Négatif \\
\hline
\end{tabular}




$\begin{array}{llllllll}\mathrm{PROXCH}^{(5)} & \text { Proximité d'un chenal } & \begin{array}{l}1 \text { si parcelle en 1ère ligne, } 0 \\ \text { sinon }\end{array} & 0 & 1 & 0,50 & 0,50 & \text { Positif }\end{array}$

a Source des données : ${ }^{(1)}$ Affaires Maritimes, ${ }^{(2)}$ ARCHYD-IFREMER, ${ }^{(3)}$ réglementation locale, ${ }^{(4)}$ réglementation européenne, ${ }^{(5)}$ auteur.

${ }^{b} L^{\prime}$ 'effet attendu sur la variable dépendante INDEM est indiqué pour une variation positive de la variable indépendante concernée. 
Distance en kilomètres au port ostréicole le plus proche (distkm). Cette distance a été mesurée à partir du sommet le plus proche de la parcelle. On peut penser que les parcelles les plus éloignées des ports sont celles qui ont une valeur moins élevée. En effet, l'activité ostréicole demande une présence régulière sur les parcs et l'éloignement des parcs peut donc être une contrainte.

Potentiel de captage en nombre équivalents-tuiles (pcapt). Cette variable tient compte de l'année de transaction, de la surface de la concession et des différentes restrictions que peuvent imposer les schémas des structures. II s'agit ici d'un potentiel, c'est-à-dire que l'on n'a pas tenu compte du fait que les parcelles étaient effectivement ou non concernées par le captage. Cela nous permettra par la suite de pouvoir estimer les effets d'une modification du schéma des structures (en termes de densité ou de surface concernée).

Salinité (sal). La salinité est généralement de 35 dans les océans tandis qu'elle est nulle dans les eaux douces (Auby, et al., 2010). Sur le Bassin d'Arcachon, elle fluctue entre 26,3 et 33,8 . Les salinités les plus élevées sont mesurées principalement à proximité des passes du Bassin d'Arcachon et les moins élevées au nord et à l'est de ce dernier. Cela est dû aux différents apports en eau douce.

Les variables suivantes ${ }^{22}$ (mes, chla, nh4 et nit) correspondent aux éléments qui influent sur la production primaire d'un milieu ${ }^{23}$. Le phytoplancton se développe grâce à la productivité primaire d'un milieu ; il sert de nourriture aux huîtres.

Quantité de matière en suspension (mes). Les matières en suspension sont des matières fines minérales insolubles qui contribuent à la turbidité de l'eau (Ropert, et al., 2007). Elles réduisent en effet la transparence de l'eau et de ce fait la production primaire photosynthétique (Manaud, et al., 1997b). Les fortes turbidités ont tendance à réduire les possibilités d'ingestion de phytoplancton (Ropert, et al., 2007). Néanmoins, selon leur nature, les matières en suspensions sont une source nutritive non négligeable (Manaud, et al., 1997b). Les matières en suspensions proviennent de rejets urbains, industriels et/ou agricoles. Leurs quantités sont donc un indicateur de la qualité de l'eau où elles sont mesurées.

Quantité de chlorophylle a (chla). La chlorophylle a est le principal pigment photorécepteur des végétaux. Le dosage de la chlorophylle a dans l'eau de mer permet une quantification de la biomasse phytoplanctonique plus simple et plus rapide que les techniques basées sur le comptage et la mesure du volume des cellules (Manaud, et al., 1997b). C'est donc un indicateur approximatif de la ressource trophique (Ropert, et al., 2007). Par ailleurs, il a été mis en évidence une relation significative entre le facteur chlorophylle $a$ et la mortalité d'huitres de la classe de 1 an (Ropert, et al., 2007). Les auteurs ont ainsi montré qu'il existe des seuils de concentration en chlorophylle a en-deçà desquels les mortalités seraient plus importantes mais également des seuils au-delà desquels les mortalités seraient également plus importantes. Les quantités de chlorophylle a sont plus importantes au nord et à l'est du Bassin d'Arcachon qu'à proximité des passes, (Manaud, et al., 1997a).

\footnotetext{
${ }^{22}$ En raison de la complexité des relations entre ces différents paramètres et la reproduction, croissance ou mortalité des huîtres, nous nous limitons à une présentation partielle de leur influence.

${ }^{23}$ On appelle productivité primaire «la quantité d'énergie chimique (composés organiques) issue de la conversion de l'énergie lumineuse par les organismes autotrophes d'un écosystème, dans une période donnée » (Campbell \& Reece, 2004).
} 
Quantité d'ammonium (NH4). L'ammonium est l'une des deux formes en solution avec l'ammoniac - de l'azote ammoniacal. Ce dernier est utilisé par les végétaux comme source d'azote. En eaux côtières non polluées et en milieu océanique, les concentrations sont généralement inférieures à $1 \mu \mathrm{mol} / \mathrm{l}$. Par contre, les concentrations sont plus élevées dans les estuaires, ce qui traduit l'influence des rejets urbains et/ou agricoles. L'ammonium est donc un bon traceur de la pollution urbaine notamment. (Manaud, et al., 1997b)

Quantité de nitrate (nit). L'ion nitrate $\left(\mathrm{NO}_{3}\right)$ est la forme oxydée stable de l'azote en solution aqueuse. II entre dans le cycle de l'azote comme support de la croissance des végétaux puis régénéré à partir des formes organiques par les bactéries. Lorsque la vitesse de régénération devient inférieure à la vitesse d'utilisation, les ions nitrate sont un facteur limitant de la croissance des algues. (Manaud, et al., 1997b)

Ces dernières variables, ainsi que celle relative à la salinité, peuvent avoir un effet positif ou négatif sur l'indemnité selon leur niveau. La comparaison des niveaux moyens de ces paramètres et des différents seuils de la Grille de lecture de la qualité générale des eaux de mer (Agences de l'eau, 2001) nous permet de dire que l'eau du Bassin d'Arcachon est globalement de bonne qualité. Cependant, cela ne nous permet pas d'anticiper sur l'effet d'une variation de ces variables sur le montant de l'indemnité, le poids de ces dernières dans l'explication du niveau de l'indemnité pouvant ou non amener à un dépassement des seuils et donc à une modification de l'appréciation de la qualité de l'eau.

Technique utilisée (tech). Deux techniques sont utilisées dans le Bassin d'Arcachon: la culture des huîtres à plat et la culture des huîtres en surélevé. L'élevage à plat consiste à répandre directement sur le sol les huîtres tandis que l'élevage en surélevé consiste à élever les huîtres dans des poches reposant sur des tables métalliques situées à 40 à $50 \mathrm{~cm}$ du $\mathrm{sol}^{24}$. Si la culture à plat nécessite moins d'investissement en matériel - pas d'utilisation de poches ostréophiles ni de tables métalliques - et moins de manutention, elle a pour inconvénient majeur d'accroître le risque d'envasement des huîtres. Egalement, les huîtres n'étant pas dans des poches, elles sont plus exposées aux prédateurs et donc les pertes sont généralement plus importantes avec cette technique qu'en élevage en surélevé. Néanmoins, cette technique a pour avantage de limiter le stress des animaux lié aux phénomènes d'exondation ${ }^{25}$ et immersion successifs, stress qui augmenterait les mortalités estivales (Maurer \& Combs, 1986).

Classement sanitaire (csanit). Le classement sanitaire des parcelles peut avoir une influence importante sur la valeur attribuée à une parcelle. En effet, une parcelle non classée A oblige l'exploitant à investir dans des installations de purification, les coquillages n'étant pas consommables immédiatement contrairement à ceux issus de parcelles classées $A$. Le classement sanitaire des parcelles fait référence à la qualité des eaux de mer. Ainsi, le Schéma de Mise en Valeur de la Mer du Bassin d'Arcachon (SMVMBA) de 2004 établit une grille de lecture quant à la qualité des

\footnotetext{
${ }^{24}$ II existe une autre technique de culture qui est l'élevage en suspension sur cordes mais cette dernière n'est pas pratiquée dans le Bassin d'Arcachon.

${ }^{25}$ L'exondation est la conséquence des phénomènes de marée et correspond à la situation de retrait de la mer.
} 
" eaux de mer ${ }^{26}$. Les concessions ostréicoles sont classées selon des zones catégorisées de $\mathrm{A}$ à $\mathrm{D}$, des zones où la qualité est la plus élevée et à celles où elle l'est le moins. Sur le Bassin d'Arcachon, la majorité des concessions sont classées en zone A. Les concessions classées en zone B sont situées principalement au nord et à l'est du Bassin.

Espèce présente sur la parcelle (espch). Nous avons distingué ici les parcelles destinées uniquement à l'huître creuse et celles qui peuvent être destinées à d'autres coquillages. Cette variable nous indique alors les possibilités de diversification de l'activité.

Utilisation de la parcelle pour l'élevage (elevdum). Cette variable a pour but de discriminer les parcelles destinées uniquement à l'élevage de celles qui ne le sont pas. En effet, le potentiel de captage présenté précédemment ne fait pas de distinction entre les parcelles qui supportent effectivement des activités d'élevage et celles qui n'en supportent pas. L'élevage se pratique dans les zones classées A. En effet, les zones classées B sont pour l'essentiel utilisées pour le captage de naissain. Le naissain n'étant pas commercialisable pour la consommation, il n'est pas nécessaire que le captage ait lieu dans des zones où le classement sanitaire correspond à des eaux de " très bonne qualité ${ }^{27}$ ".

Proximité des chenaux (proxch). Cette variable a pour but de voir quel est l'influence de la proximité d'une parcelle à un chenal. En effet, cette proximité permet d'une part à l'ostréiculteur d'y accéder plus aisément mais également, elle permet aux huîtres élevées sur ces parcs de bénéficier davantage des différents apports nutritifs par exemple. Enfin, la proximité d'un chenal a pour effet de diminuer la durée d'exondation de la parcelle.

\subsection{Spécification et détermination du modèle spatial de prix hédoniques ${ }^{28}$}

La forme fonctionnelle déterminée à partir de la transformation Box-Cox (Box \& Cox, $1964)$ est une forme $\log -\log ^{29}$. Afin de confirmer l'adéquation de cette forme fonctionnelle à notre modèle, nous avons estimé les différentes formes fonctionnelles et appliqué le test RESET ${ }^{30}$ (Ramsey, 1969). Nous comparons également les $\mathrm{RMSE}^{31}$ ainsi que le pouvoir prédictif du modèle à partir du $\mathrm{R}^{2}$. Les résultats (Tableau 2) montrent que l'hypothèse $H_{0}$ selon laquelle le modèle est correctement spécifié peut être retenue uniquement dans le cas de la forme fonctionnelle log-log qui par ailleurs présente le RMSE le plus faible et le $\mathrm{R}^{2}$ le plus élevé.

\footnotetext{
${ }^{26}$ Cette grille de salubrité des zones de production conchylicole a été établie sur la base du décret $n^{\circ} 94.340$ du 28 avril 1994 relatif aux conditions sanitaires de production et de mise sur le marché des coquillages vivants.

${ }^{27}$ Par « très bonne qualité » nous faisons ici référence au fait que dans les zones classées $A$, les huîtres élevées peuvent être directement consommées.

${ }^{28}$ Les estimations et les tests statistiques ont été réalisés sous STATA et Matlab.

${ }^{29}$ La valeur du paramètre de Box-Cox pour une transformation identique des deux termes de l'équation est égale à 0,10 . Cette valeur très proche de 0 indique alors que la forme fonctionnelle adéquate est une double-log.

${ }^{30}$ Ramsey Regression Equation Specification Error Test.

${ }^{31}$ RMSE pour Root Mean Squared Error qui mesure l'erreur totale d'estimation des modèles. Le modèle à retenir est alors celui dont la valeur du RMSE est la plus faible.
} 
Tableau 2. Comparaison des différentes formes fonctionnelles

\begin{tabular}{lcccc} 
& Linéaire & Log-linéaire & Linéaire-log & Double log \\
\hline Test RESET & 6,5 & 6,58 & 14,98 & 0,62 \\
RMSE & $(0,0003)$ & $(0,0002)$ & $(0,0000)$ & $(0,6025)$ \\
$\mathrm{R}^{2}$ & 4875,8 & 0,84 & 4899,5 & 0,74 \\
\hline
\end{tabular}

Ainsi, le modèle à estimer est de la forme suivante :

$$
\ln Y=\alpha+\beta \ln X+\phi Z+\varepsilon
$$

Une fois déterminé la forme fonctionnelle, nous avons procédé dans un premier temps à l'estimation du modèle aspatial à l'aide de la méthode des Moindres Carrés Ordinaires $^{32}$ afin d'appliquer les différents tests de spécification et d'autocorrélation spatiale et pouvoir déterminer ainsi le type de modèle économétrique spatial devant être utilisé. Les résultats sont donnés dans le Tableau 3.

Tableau 3. Tests de spécification et de dépendance spatiale

Test

Statistique

Probabilité

\begin{tabular}{lrl}
\hline Spécification & & \\
\hline Jarque-Bera & 29,612 & 0,000 \\
JLM test & 65,190 & $1,41^{\mathrm{e}-008}$ \\
Koenker-Bassett & 18,970 & 0,124 \\
\hline Diagnostic de l'autocorrélation spatiale & & \\
\hline I de Moran & 7,869 & 0,000 \\
$L M_{L A G}$ & 34,798 & $3,66^{\mathrm{e}-009}$ \\
$L M_{E R R}$ & 46,220 & $1,06^{\mathrm{e}-011}$ \\
$R L M_{L A G}$ & 2,805 & 0,094 \\
$R L M_{E R R}$ & 14,227 & $1,62^{\mathrm{e}-004}$ \\
$L M_{B I}$ & 49,024 & $2,26^{\mathrm{e}-011}$ \\
\hline
\end{tabular}

Le test de Jarque-Bera (Jarque \& Bera, 1987) rejette l'hypothèse nulle de normalité des résidus. Nous procédons ensuite au test joint d'autocorrélation spatiale et d'hétéroscédasticité (JLM). Ce dernier étant significatif, nous testons alors

\footnotetext{
${ }^{32}$ Les résultats de cette estimation sont indiqués dans le Tableau 6.
} 
uniquement la présence d'hétéroscédasticité grâce au test de Koenker-Bassett (1982) qui est robuste à la non-normalité des résidus. L'hypothèse nulle d'homoscédasticité est acceptée. La significativité du test joint est donc liée à la présence uniquement d'autocorrélation spatiale. De la même façon, le test de Moran confirme le test JLM selon lequel il existe une autocorrélation spatiale.

Afin de déterminer le modèle spatial le plus adapté, nous comparons les différents tests du multiplicateur de Lagrange et leurs versions robustes. Ainsi, selon la règle de décision proposée par Anselin et Rey (1991), Florax et Folmer (1992), Anselin et Florax (1995) et Anselin, et al. (1996), nous rejetons le modèle spatial autorégressif (SAR), le $L M_{E R R}$ étant plus significatif que le $L M_{L A G}$ et le $R L M_{E R R}$ étant significatif mais pas le $R L M_{L A G}$. De plus, le $L M_{B I}$ nous conduit à rejeter l'hypothèse nulle, le modèle spatial général (SAC) n'est donc pas adapté. Enfin, pour déterminer entre le modèle Durbin spatial (SDM) et le modèle avec autocorrélation spatiale des erreurs (SEM) celui qui est le plus approprié, nous utilisons les critères d'informations d'Akaïke (AIC) et de Schwarz (SC). Les valeurs de ces deux critères $^{33}$ nous permettent de justifier le choix d'un modèle avec autocorrélation spatiale des erreurs, ce dernier minimisant à la fois l'AIC et le SC.

Nous avons ensuite contrôlé l'absence d'endogénéité des variables indépendantes ${ }^{34}$. Le Tableau 4 reprend les résultats des différents tests. Les statistiques d'Hausman et Sargan ${ }^{35}$ étant toutes inférieures au seuil de $5 \%$, nous pouvons conclure à l'exogénéité de l'ensemble des variables indépendantes continues de notre modèle.

\footnotetext{
${ }^{33}$ Elles sont respectivement de 615 et 724 pour le modèle SDM et de 612 et 669 pour le modèle SEM.

${ }^{34}$ On distingue généralement trois sources principales d'endogénéité : une erreur de mesure sur la variable dépendante, une simultanéité qui correspond au fait que certaines variables explicatives et la variable expliquée sont déterminées « en même temps » ou alors l'omission de variables explicatives. Dans les études foncières classiques, un problème d'endogénéité se pose systématiquement lorsqu'une variable relative à la surface est introduite. Ce problème ne se pose pas dans notre cas puisque la surface a été introduite de façon indirecte, dans la construction de la variable relative au potentiel de captage. Toutefois, la rigueur nous impose de vérifier l'exogénéité de l'ensemble des variables indépendantes.

${ }^{35}$ Le test de Sargan (Sargan, 1958) est ici préféré à celui d'Hansen (Hansen, 1982), ce dernier s'utilisant préférentiellement en présence d'hétéroscédasticité.
} 


\begin{tabular}{|c|c|c|c|c|}
\hline \multicolumn{5}{|c|}{$\begin{array}{l}\text { Tableau 4. Résultats des tests d'endogénéité et de validité des } \\
\text { instruments }\end{array}$} \\
\hline \multirow[b]{2}{*}{ Instruments } & \multicolumn{2}{|c|}{$\begin{array}{l}\text { Variables non } \\
\text { transformées }\end{array}$} & \multicolumn{2}{|c|}{ Variables transformées } \\
\hline & $\begin{array}{l}\text { Test } \\
\text { d'Haus } \\
\text { man }\end{array}$ & $\begin{array}{c}\text { Test de } \\
\text { Sarg } \\
\text { an }\end{array}$ & $\begin{array}{l}\text { Test } \\
\text { d'Haus } \\
\text { man }\end{array}$ & $\begin{array}{c}\text { Test de } \\
\text { Sarg } \\
\text { an }\end{array}$ \\
\hline INST_NBT_1 & $\begin{array}{c}0,155 \\
(0,6936)\end{array}$ & $\begin{array}{c}0,207 \\
(0,6493)\end{array}$ & $\begin{array}{c}0,455 \\
(0,5001)\end{array}$ & $\begin{array}{c}0,019 \\
(0,8911)\end{array}$ \\
\hline INST_DISTKM & $\begin{array}{c}0,374 \\
(0,5408)\end{array}$ & $\begin{array}{c}0,007 \\
(0,9335)\end{array}$ & $\begin{array}{c}0,136 \\
(0,7125)\end{array}$ & $\begin{array}{c}2,094 \\
(0,1479)\end{array}$ \\
\hline INST_CAPT & $\begin{array}{c}1,813 \\
(0,1781)\end{array}$ & $\begin{array}{c}0,261 \\
(0,6093)\end{array}$ & $\begin{array}{c}1,502 \\
(0,2204)\end{array}$ & $\begin{array}{c}0,000 \\
(0,9950)\end{array}$ \\
\hline INST_SAL & $\begin{array}{c}1,073 \\
(0,3003)\end{array}$ & $\begin{array}{c}0,301 \\
(0,5832)\end{array}$ & $\begin{array}{c}0,122 \\
(0,7273)\end{array}$ & $\begin{array}{c}0,002 \\
(0,9662)\end{array}$ \\
\hline INST_MES & $\begin{array}{c}0,043 \\
(0,8352)\end{array}$ & $\begin{array}{c}0,499 \\
(0,4799)\end{array}$ & $\begin{array}{c}0,547 \\
(0,4596)\end{array}$ & $\begin{array}{c}1,150 \\
(0,2835)\end{array}$ \\
\hline INST_CHLA & $\begin{array}{c}0,041 \\
(0,8400)\end{array}$ & $\begin{array}{c}0,401 \\
(0,5265)\end{array}$ & $\begin{array}{c}0,445 \\
(0,5049)\end{array}$ & $\begin{array}{c}2,687 \\
(0,1012)\end{array}$ \\
\hline INST_NH4 & $\begin{array}{c}0,035 \\
(0,8511)\end{array}$ & $\begin{array}{c}2,124 \\
(0,1450)\end{array}$ & $\begin{array}{c}0,037 \\
(0,8473)\end{array}$ & $\begin{array}{c}0,991 \\
(0,3196)\end{array}$ \\
\hline INST_NIT & $\begin{array}{c}2,406 \\
(0,1209)\end{array}$ & $\begin{array}{c}0,458 \\
(0,4983)\end{array}$ & $\begin{array}{c}0,200 \\
(0,6545)\end{array}$ & $\begin{array}{c}0,356 \\
(0,5507)\end{array}$ \\
\hline $\begin{array}{l}\text { LM error } \\
\text { Test }\end{array}$ & $\begin{array}{l}46 \\
10,(\end{array}$ & & (0, & \\
\hline
\end{tabular}

Note : La méthode utilisée ici est celle des variables instrumentales. Les instruments utilisés pour détecter la présence d'endogénéité sont des quasi-instruments correspondant à des classes de valeurs des variables suspectées d'endogénéité.

Les propriétés du test de Sargan pouvant être affectées en présence d'autocorrélation spatiale, nous réalisons ce dernier à la fois sur le modèle $M C O$ (variables non transformées) et à partir de variables ayant été transformées par la méthode de Cochrane-Orcutt (Cochrane \& Orcutt, 1949). La variable transformée $\left(X^{T}\right)$ est alors obtenue à partir de la variable non transformée $(X)$ comme suit : $X^{T}=X .(I-\lambda W)$ avec une matrice identité, $W$ la matrice de poids et $\lambda$ le paramètre reflétant l'intensité de l'interdépendance entre les résidus et le terme d'erreur. Cette transformation est appliquée à l'ensemble des variables endogène et exogènes et permet d'obtenir des variables spatialement filtrées, sans autocorrélation spatiale. Cette procédure est réalisable dans notre cas en raison de la présence d'autocorrélation spatiale uniquement dans le terme d'erreur comme cela a été mis en évidence par les différents tests de spécification. Les p-value des tests sont données entre parenthèses. 
Comme pour l'analyse exploratoire de données spatiales, nous utilisons une matrice de poids des 8 plus proches voisins pour l'estimation du modèle de prix hédoniques avec autocorrélation spatiale des erreurs. Les résultats de cette estimation sont les suivants (Tableau 5).

\begin{tabular}{|c|c|c|c|c|c|}
\hline \multirow{2}{*}{ Variables } & \multirow{2}{*}{ VIF } & \multicolumn{2}{|c|}{$\mathrm{MCO}$} & \multicolumn{2}{|l|}{ SEM } \\
\hline & & $\beta$ & t-stat & $\beta$ & t-stat \\
\hline CONSTANTE & & $-40,1198 * * *$ & $-7,04$ & $-33,3361^{* * *}$ & $-5,26$ \\
\hline NBT_1 & 2,87 & $-0,4622$ *** & $-3,91$ & $-0,5134^{* * *}$ & $-4,55$ \\
\hline DISTKM & 1,71 & $-0,2379 * * *$ & $-4,02$ & $-0,2749 * * *$ & $-3,34$ \\
\hline CAPT & 1,55 & $0,8265^{* * *}$ & 16,39 & $0,8407^{* * *}$ & 17,29 \\
\hline SAL & 2,47 & 11,5569 *** & 7,36 & $9,6415^{* * *}$ & 5,44 \\
\hline MES & 2,13 & $0,9224 * * *$ & 4,05 & $0,9258 * * *$ & 4,02 \\
\hline CHLA & 2,21 & $-0,3332$ * & $-1,98$ & $-0,5503^{* *}$ & $-3,11$ \\
\hline $\mathrm{NH} 4$ & 1,80 & 0,2132 * & 2,22 & $0,3230 * *$ & 3,26 \\
\hline NIT & 3,00 & $0,2107^{* * *}$ & 4,42 & $0,2102 * * *$ & 4,56 \\
\hline $\mathrm{TECH}$ & 1,28 & $-0,2621^{* *}$ & $-2,79$ & $-0,1897$ * & $-2,08$ \\
\hline CSANIT & 1,68 & 0,3859 * & 2,47 & 0,4648 * & 2,31 \\
\hline ELEVDUM & 2,06 & $0,5406^{* * *}$ & 4,30 & $0,3506^{* *}$ & 2,82 \\
\hline ESPHC & 1,24 & $-0,3370 * * *$ & $-3,99$ & $-0,2539 * *$ & $-3,01$ \\
\hline PROXCH & 1,24 & 0,2188 ** & 2,68 & $0,1512 \dagger$ & 1,92 \\
\hline Lambda $(\lambda)$ & & & & $0,4650 * * *$ & 6,87 \\
\hline$R^{2}$ & & 0,58 & & 0,6332 & \\
\hline
\end{tabular}

Niveaux de significativité : $† p<0,1{ }^{*} p<0,05{ }^{* *} p<0,01{ }^{* * *} p<0,001$

Le modèle avec autocorrélation spatiale des erreurs explique un peu plus de $63 \%$ des indemnités de substitution des concessions ostréicoles arcachonnaises. La constante de ce modèle est significative au seuil de $0,1 \%$ ce qui suggère l'existence de variables explicatives non incluses dans le modèle. En effet, des variables telles que l'état des parcelles (équipement, ensablement par exemple) auraient sans doute amélioré le pouvoir explicatif du modèle, ces informations pouvant influencer la productivité des parcelles. De la même façon, des variables relatives aux exploitations des concessionnaires cédants et repreneurs (comme la taille de l'exploitation ou encore la localisation des autres concessions détenues) auraient permis d'affiner davantage notre analyse. 
Concernant le paramètre $\lambda$ qui désigne l'intensité de la dépendance spatiale entre les résidus, sa significativité au seuil de $0,1 \%$ et son signe indique l'existence d'une autocorrélation spatiale positive dans la distribution des indemnités de substitution et confirme les résultats obtenus lors de l'ESDA effectuée précédemment.

\section{Interprétation des résultats}

Les résultats de l'estimation de ce modèle indiquent que les variables explicatives peuvent être regroupées selon qu'elles influent positivement ou négativement sur le montant de l'indemnité de substitution à mesure que leur valeur augmente (Figure 4).

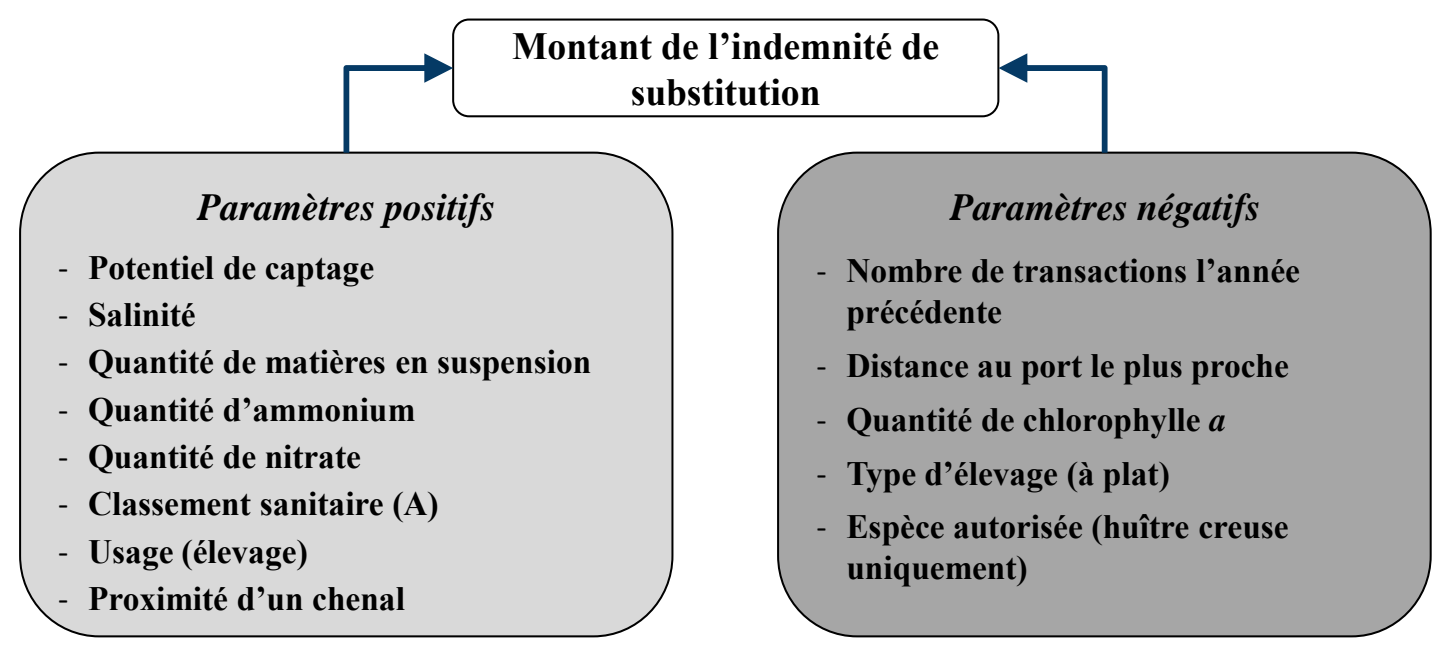

Figure 4. Regroupement des variables selon leur influence sur l'indemnité de substitution

S'agissant de la disponibilité des parcelles, les résultats montrent qu'un nombre important de transactions l'année précédente a pour effet de diminuer le montant de l'indemnité de substitution. Cela est cohérent avec la logique de marché faisant dépendre l'indemnité de substitution des ajustements entre l'offre et la demande mais également de la situation économique du secteur (Mongruel, et al., 2008b). Si nous ne sommes pas en mesure d'expliquer le sens des ajustements (hausse de la demande plus forte que celle de l'offre ou baisse de la demande plus forte que celle de l'offre), certaines explications peuvent être avancées pour les années où les transactions ont été les plus nombreuses $(2001,1996)$ et les moins nombreuses (2007, 1992 et 1988). Ainsi, l'année 2007, qui correspond à celle où il y a eu le moins de transactions, fait suite à celle où les fermetures administratives ont commencé à devenir de plus en plus fréquentes. II est donc tout à fait possible que les entreprises n'aient pas souhaité acquérir de nouvelles parcelles, leur situation financière s'étant fortement dégradée en 2006. Ensuite, 1992 correspond à la modification du schéma des structures qui instaure des règles d'exploitation différentes selon la localisation des concessions. Les dispositions introduites ont eu pour conséquence de diminuer la biomasse en élevage et le potentiel de captage des exploitations ; cela a sans doute créé un besoin en surfaces supérieur à celui qu'il était précédemment afin de maintenir le niveau de production, pouvant expliquer une faiblesse de l'offre. Enfin, 1988 fait suite à l'officialisation des transactions marchandes et à l'adoption du $1^{\text {er }}$ schéma des structures, ce qui a pu 
entraîner une hausse de l'offre plus importante que celle de la demande. S'agissant des années où les transactions ont été les plus nombreuses, l'année 2001 coïncide avec celle de la modification du schéma des structures (au mois d'avril) faisant passer la taille minimale de référence de 1,2 hectares à 90 ares pouvant expliquer une hausse de l'offre, certaines parcelles conservées jusque là uniquement afin de respecter la taille de référence étant devenues "inutiles". La modification intervenue en 2001 instaure également une dimension minimale de $1^{\text {ère }}$ installation ce qui a pu avoir comme conséquence un développement des attributions gratuites dans les mois suivants. Cela se confirme d'ailleurs lorsque l'on compare le nombre mensuel moyen de transaction avant et après le mois d'avril. On est passé en effet à une moyenne mensuelle de 20 transactions avant le mois d'avril à un peu plus de 30 à partir du mois de mai. Enfin, 1996 fait suite à deux années de captage pléthorique (Auby \& Maurer, 2004) qui ont pu avoir pour conséquence une hausse de la demande supérieure à celle de l'offre. II est donc probable qu'il y ait un effet temporel décalé sur le prix des concessions et cela est d'autant plus envisageable que le montant des indemnités de substitution est censé être cohérent avec le prix moyen des indemnités des transactions des années précédentes.

Concernant l'accessibilité, une parcelle à proximité immédiate d'un chenal sera plus valorisée qu'une parcelle de deuxième ou troisième ligne, une parcelle de première ligne étant plus accessible. Cela est également vrai s'agissant de la distance au port le plus proche. Plus une parcelle sera éloignée d'un port et moins elle sera valorisée. Si la proximité d'un port réduit les coûts liés au transport tout comme la proximité d'un chenal, la proximité de ce dernier a pour autre avantage de réduire les temps d'exondation et donc de diminuer le stress des animaux. Cette valorisation de la diminution du stress aurait également pu être révélée avec la variable relative à la technique utilisée puisque l'élevage à plat réduit les temps d'exondation et donc les temps de croissance des animaux voire leurs mortalités. Mais cette réduction est sans doute moins importante que les mortalités plus élevées qui sont généralement observées dans les élevages à plat et qui sont dues principalement aux prédateurs.

La réglementation est déterminante du montant de l'indemnité de substitution. En effet, une concession avec un potentiel de captage élevé sera davantage valorisée qu'une parcelle avec un potentiel plus faible. De la même façon, une parcelle destinée à l'élevage sera associée à une indemnité plus élevée que si cette même parcelle est destinée au captage et cela davantage encore si l'élevage est effectué en surélevé et non à plat.

S'agissant des paramètres hydrologiques, le niveau de salinité est positivement relié au montant de l'indemnité de substitution. Cela s'explique par le fait que l'on trouve les salinités les plus élevées à proximité des passes du bassin (à l'entrée du bassin), région qui est considérée comme la plus productive grâce aux apports océaniques. Enfin, en ce qui concerne les concentrations en matières en suspension, en ammonium et en nitrates, plus elles sont élevées et plus la parcelle est valorisée car considérée comme située dans une eau de bonne qualité ${ }^{36}$ contrairement à une eau présentant des concentrations élevées en chlorophylle a. En effet, au-delà d'un certain seuil, des concentrations élevées en chlorophylle a sont considérées comme un indicateur de mauvaise qualité des eaux.

${ }^{36}$ Cela est vrai dans le Bassin d'Arcachon parce que les quantités de matières en suspension, d'ammonium et de nitrates sont peu élevées. Une augmentation de la quantité de ces éléments contribue alors à améliorer la croissance des huîtres. Cependant, à partir d'un certain seuil correspondant à des quantités 4 à 5 fois supérieures aux quantités relevées jusqu'à présent, l'eau sera jugée de mauvaise qualité. 
A partir des résultats de l'estimation, il nous est alors possible de calculer les prix marginaux ${ }^{37}$ de chacune des composantes des indemnités de substitution étudiées. En considérant une indemnité de substitution moyenne de $5604 €$, les prix marginaux sont alors les suivants (Tableau 6).

\begin{tabular}{lcc}
\hline \multicolumn{3}{l}{ Tableau 6. Décomposition de l'indemnité moyenne de substitution } \\
\hline Paramètres & $\begin{array}{c}\text { Prix marginal } \\
\text { implicite }(€)\end{array}$ & $\begin{array}{c}\text { \% de variation } \\
\text { de l'indemnité }\end{array}$ \\
\hline Nombre de transactions l'année précédente & -42 & $-0,8$ \\
Distance au port le plus proche $^{\text {b }}$ & -75 & $-1,3$ \\
Potentiel de captage $^{c}$ & 276 & 4,9 \\
Salinité & 1688 & 30,1 \\
Matières en suspension & 855 & 15,3 \\
Chlorophylle a & -1685 & $-30,1$ \\
Ammonium & 739 & 13,2 \\
Nitrate & 219 & 3,9 \\
Technique de production autorisée & -968 & $-17,3$ \\
Classement sanitaire & 3316 & 42,0 \\
Utilisation de la parcelle & 2353 & 46,5 \\
Espèce élevée & -1257 & $-22,4$ \\
Proximité d'un chenal & 914 & 16,3 \\
\hline
\end{tabular}

all s'agit ici du pourcentage de variation du montant de l'indemnité moyenne suite à une variation de $1 \%$ du niveau de la variable explicative concernées, toutes choses égales par ailleurs (hors cas particuliers de la distance au port le plus proche et du potentiel de captage pour lesquels les variations considérées sont les mêmes que celles ayant permis de déterminer le prix marginal implicite).

${ }^{b}$ Le prix marginal implicite correspondant à la distance au port le plus proche est calculé pour une variation de 100 mètres et non d'un kilomètre.

'Le prix marginal implicite correspondant au potentiel de captage est calculé pour une variation de 1000 équivalents-tuiles. Cette variation peut correspondre à une augmentation de la surface concernée par le captage en moyenne de 0,89 ares (la surface concernée passant alors de 13,34 ares en moyenne à 14,23 ares en moyenne) ou encore à une augmentation de la densité moyenne à l'are de 238 équivalents-tuiles (la densité moyenne à l'are passant alors de 1534 à 1772 équivalents-tuiles).

Ce tableau nous indique que l'indemnité de substitution moyenne est expliquée principalement par le classement sanitaire en zone A de la parcelle et son utilisation pour l'élevage. Une parcelle sera alors payée $2353 €$ plus cher en moyenne si l'élevage peut y être pratiqué et $3316 €$ de plus si elle est en zone A. Par contre, la quantité de chlorophylle a et l'espèce pouvant être élevée sont des paramètres dont le poids est important dans l'indemnité de substitution moyenne mais ce poids est

\footnotetext{
${ }^{37}$ Les méthodes de calcul des consentements marginaux à payer sont en annexe.
} 
négatif. Ainsi, une parcelle sera échangée $1685 €$ de moins si les concentrations en chlorophylle a sont élevées et $1257 €$ de moins s'il ne peut y être élevé que des huîtres creuses.

A partir de ces prix implicites, on peut alors déterminer le poids de chaque type de composante dans l'indemnité moyenne. Ainsi, les composantes liées à la réglementation et à la productivité naturelle représentent près de $90 \%$ du prix moyen. Les variables liées à la productivité naturelle (salinité, matières en suspension, chlorophylle $a$, ammonium, nitrates) représentent près d'un quart de l'indemnité moyenne. Une variation de $1 \%$ de l'ensemble de ces paramètres a pour conséquence d'augmenter l'indemnité moyenne d'un peu plus de $32 \%$. Par contre, environ deux tiers de l'indemnité moyenne sont expliqués par des variables liées à la réglementation (le potentiel de captage, la technique, l'utilisation et l'espèce autorisées, le classement sanitaire). On voit donc bien ici le rôle primordial de la réglementation qui contribue au maintien d'un niveau de productivité satisfaisant pour pérenniser à court et moyen terme l'activités ${ }^{38}$, permettant que le délai d'obtention d'une huître creuse commercialisable pour la consommation à partir d'un naissain ne dépasse généralement pas les trois ans.

\section{Conclusion}

A partir d'un modèle de prix hédoniques tenant compte de la localisation des parcelles ostréicoles, nous avons mis en évidence l'importance de la localisation des concessions dans la formation du prix d'échange de ces concessions. Un résultat intéressant de cette modélisation est l'influence de l'éloignement des parcelles aux ports. En effet, ce résultat est conforme à la théorie de la localisation de Von Thünen (1826) telle que présentée par Beckmann (1999). Ainsi, les ostréiculteurs bénéficient d'une rente différentielle de situation égale à l'économie de coût de transport en se localisant sur les parcelles les plus proches des ports. Cette rente décroit à mesure de l'éloignement des concessions par rapport aux ports. L'importance de la localisation dans la valorisation des concessions est aussi liée à la productivité du milieu. En effet, les parcelles situées à l'est ou au nord du Bassin sont généralement moins valorisées que celles situées au sud du Bassin, à proximité des passes en raison de différences de productivité naturelle. Le sud du Bassin présente des conditions de salinité, de température, de disponibilité en ressources trophiques et plus généralement une qualité environnementale meilleure que dans le reste du Bassin. Cela explique par ailleurs que les concessions localisées à l'est du Bassin soient utilisées essentiellement pour le captage car cette activité ne requiert pas un niveau de qualité de l'eau aussi important que pour l'élevage. Cela justifie également que les concessions situées dans les zones les plus productives soient soumises à des contraintes réglementaires d'exploitation et de densité d'animaux en élevage plus strictes afin de limiter les risques de surcharge des parcs qui auraient pour conséquence de dégrader les conditions de production comme cela a pu être le cas par le passé, où le cycle de production était passé à 5 ans avec des huîtres de moins bonne qualité ; cela était lié essentiellement aux densités en élevage ayant conduit à une surexploitation de la capacité biotique du milieu. En l'absence de régulation, cette dégradation des conditions de production se manifesterait donc par une baisse de la productivité des parcelles et la valeur de ces dernières diminuerait

\footnotetext{
${ }^{38}$ II est probable en effet que la réglementation actuelle soit amenée à être modifiée afin de tenir compte de l'évolution des conditions environnementales de production en lien notamment avec le changement global.
} 
à mesure qu'elles seraient exploitées. Néanmoins, les pouvoirs publics ne doivent pas se contenter de réguler l'utilisation du Domaine Public Maritime par les seuls ostréiculteurs. Ils se doivent également de considérer les usages concurrents de l'ostréiculture afin que chaque utilisateur intègre la composante de bien public du Bassin d'Arcachon. Cela passe alors par une maîtrise de l'urbanisation, par un encadrement du développement de la plaisance, etc., et nécessite donc une approche en terme de Gestion Intégrée des Zones Côtières.

\section{Références}

Anonyme. 2011. Recensement des entreprises ostréicoles, caractérisation de la situation des conjoints et évaluation des besoins de formation. Document de travail du Comité Régional de la Conchyliculture d'Arcachon Aquitaine.

Agences de l'Eau. 2001. Définition d'un système d'évaluation de la qualité (SEQ) des milieux littoraux. Rapport final, Réf : I090-RTOR-02/2000.

Anselin, L. 1988. Spatial econometrics: methods and models. Kluwer Academic Publishers, Dordrecht, The Netherlands.

Anselin, L. 1995a. Exploring Spatial Data with GeoDa: A Workbook. Center for Spatial Integrated Social Science, Santa Barbara.

Anselin, L. 1995b. Local indicators of spatial association - LISA. Geographical Analysis, 27 (2): 93-115.

Anselin, L. 1996. The Moran scatterplot as an ESDA tool to assess local instability in spatial association. In Spatial Analitycal Perspectives on GIS edited by Fischer M., Scholten H.J., Unwin D., pp. 11-125. Taylor \& Francis, London.

Anselin, L. 1999. Spatial econometrics. Université du Texas, Dallas.

Anselin, L., \& Bao, S. 1997. Exploratory spatial data analysis linking Spacestat and Arcview, In Recent Developments in Spatial Analysis: Spatial Statistics, Behavioural Modelling and Computational Intelligence edited by Fischer M. M. \& Gestis A., pp. 35-59. Springer-Verlag, Berlin, New York.

Anselin, L., \& Florax, R. 1995. Small sample properties of tests for spatial dependence in regression models. In New Directions in Spatial Econometrics edited by Anselin L. \& Florax R., pp.21-74. Springer.

Anselin, L., \& Rey, S. 1991. Properties of tests for spatial dependence in linear regression models. Geographical Analysis, 23: 112-131.

Anselin, L., Bera, A. K., Florax, R., \& Yoon, M. J. 1996. Simple diagnostic tests for spatial dependence. Regional Science and Urban Economics, 26 (1): 77-104.

Auby, I., Neaud-Masson, N., \& Trut, G. 2010. Bulletin de surveillance de la Qualité du Milieu Marin Littoral. Départements Gironde, Landes et Pyrénées Atlantiques. Ifremer/RST.LER/AR/10.003/Laboratoire Environnement et Ressources d'Arcachon.

Beckmann, M. J. 1999. Lectures on location theory. Springer. 
Box, G. E., \& Cox, D. R. 1964. An analysis of transformations. Journal of the Royal Society. Series B (Methodological), 26 (2): 211-252.

Campbell, N. A., \& Reece, J. B. 2004. Biologie. Adaptation et révision scientifique de R. Mathieu. De Boeck.

Cliff, A. D., \& Ord, J. K. 1981. Spatial processes: models \& applications. Pion, London.

Cochrane, D., \& Orcutt, G. H. 1949. Application of least squares regression to relationships containing auto-correlated error terms. Journal of the American Statistical Association, 44 (245): 32-61.

Dall'Erba, S. 2004. Les politiques de développement régional en Europe à la lumière des outils récents de la science régionale. Thèse de Doctorat ès Sciences Economiques. Pau : Université de Pau et des Pays de l'Adour.

Dumont, P. 1986. Analyse de l'ostréiculture : marché, production, utilisation du littoral. Thèse de Doctorat en Sciences Agronomiques. Rennes, Ecole Nationale Supérieure Agronomique de Rennes.

Fingleton, B., \& Le Gallo, J. 2008a. Finite sample properties of estimators of spatial models with autoregressive, moving average, disturbances and system feedback. Annals of Economics and Statistics: Spatial Econometrics, Innovative Networks and Growth, 87/88: 39-62.

Fingleton, B., \& Le Gallo, J. 2008b. Estimating spatial models with endogenous variables, a spatial lag and spatially dependent disturbances: finite sample properties. Papers in Regional Science, 87 (3): 319-339.

Florax, R., \& Folmer, H. 1992. Specification and estimation of spatial linear regression models. Regional Science and Urban Economics, 22: 405-432.

Hansen, L. 1982. Large sample properties of generalized method of moments estimators. Econometrica, 50 (3): 1029-1054.

Hardin, G. 1968. The tragedy of the commons. Science, 162: 1243-1248.

Héral, M., Delous-Paoli, J.-M., \& Prou, J. 1986. Dynamique des productions et des biomasses des huîtres creuses cultivées (Crassostrea Angulata et Crassostrea Gigas) dans le bassin de Marennes-Oléron depuis un siècle. CIEM - Conseil International pour l'Exploration de la Mer.

Irichabeau, G. 2011. Evaluation économique de la dépendance d'une activité au milieu naturel. L'exemple de l'ostréiculture arcachonnaise. Thèse de Doctorat ès Sciences Economiques. Bordeaux : Université Montesquieu - Bordeaux IV.

Jarque, C. M., \& Bera, A. K. 1987. A test for normality of observations and regression residuals. International Statistical Review, 55 (2): 163-172.

Kim, C. W., Phipps, T. T., \& Anselin, L. 2003. Measuring the benefits of air quality improvment: a spatial hedonic approach. Journal of Environmental Economics and Management, 45 (1): 24-39. 
Koenker, R., \& Bassett, G. 1982. Robust tests for heteroskedasticity based on regression quantiles. Econometrica, 50 (1): 43-61.

Le Gallo, J. 2002. Econométrie spatiale : l'autocorrélation spatiale dans les modèles de régression linéaire. Economie et Prévision, 155: 139-157.

Le Gallo, J. 2004. Hétérogénéité spatiale : principes et méthodes. Economie et Prévision, 162: 151-172.

LeSage, J. P. 1999. The theory and practice of spacial econometrics. Université de Toledo, $309 \mathrm{p}$.

Manaud, F., Bouchet, J.-M., Deltreil, J.-P., Maurer, D., Trut, G., Auby, I., Dreno J.P., L'Yavanc J., Masson N., \& Pellier C. 1997a. Etude intégrée du Bassin d'Arcachon Tome 2 : Qualité des eaux et des sédiments. Rapport Interne DEL/Arcachon.

Manaud, F., Bouchet, J.-M., Deltreil, J.-P., Maurer, D., Trut, G., Auby, I., Dreno J.P., L'Yavanc J., Masson N., \& Pellier C. 1997b. Etude intégrée du Bassin d'Arcachon Tome 5 : Activités ressources vivantes. Rapport Interne DEL/Arcachon.

Maurer, D., \& Combs, M. 1986. Mortalités estivales de I'huître Crassostrea Gigas dans le Bassin d'Arcachon : facteurs de milieu, aspects biochimiques et histologiques. In Pathology In Marine Aquaculture (Special publication $n^{\circ} 9$ ) edited by C. P. Vivares, J. R. Bonami \& E. Jaspers, pp. 29-41. European Aquaculture Society, Bredene Belgium.

Mongruel, R., Perez Agundez, J. A., \& Girard, S. 2008a. Droits à produire transférables : le marché des concessions conchylicoles et ses effets non désirés. Economie Rurale 306: 23-38.

Mongruel, R., Perez Agundez, J. A., Girard, S., Bailly, D., \& Nassiri, A. 2008b. Analyse des stratégies d'occupation du domaine maritime côtier : le cas des concessions conchylicoles. Rapport final du projet ASTRODOME.

Moran, P. 1948. The interpretation of statistical maps. Journal of the Royal Statistical Society. Series B (Methodological), 10 (2): 243-251.

Moran, P. 1950. A test for the serial independence of residuals. Biometrika, 37 (1/2): 178-181.

Mueller, J. M., \& Loomis, J. B. 2008. Spatial dependence in hedonic property models: do different corrections for spatial dependence result in economically significant differences in estimated implicit prices? Journal of Agricultural and Resource Economics, 33 (2): 212-231.

Palmquist, R. B. 1989. Land as differenciated factor of production: A hedonic model and its implications for welfare measurement. Land Economics, 65 (1): 23-28.

Ramsey, J-B. 1969. Tests for specification errors in classical linear least-squares regression analysis. Journal of American Statistical Association, 69: 935-939.

Ricardo, D. 1819. Des Principes de l'Economie Politique et de I'Impôt. Paris: Traduit de l'anglais par F. S. Constancio. Tome Premier. 
Ridker, R. G., \& Henning, J. A. 1967. The determinants of residential propoerty values with special reference to air pollution. The Review of Economics and Statistics, 49 (2): 246-257.

Ropert, M., Mazurié, J., Bédier, E., Le Coz, F., \& Soletchnik, P. 2007. Evaluation des risques dans les écosystèmes conchylicoles. In Mortalités estivales de l'huître creuse Crassostrea Gigas, Défi Morest edited by J.-F. Saimain, \& H. McCombie, pp. 1-49. Ifremer/Quæ.

Rosen, S. 1974. Hedonic prices and implicit markets: product differenciation in pure competition. The Journal of Political Economy, 82 (1): 34-55.

Sargan, J. D. 1958. The estiamtion of economic relationships using instrumental variables. Econometrica, 26 (3): 393-415.

Terra, S. (2005). Guide des bonnes pratiques pour la mise en oeuvre de la méthode des prix hédoniques. Ministère de l'Ecologie et du Développement Durable, Direction des Etudes Economiques et de l'Evaluation Environnementale.

Von Thünen, J. H. 1826. Der isolirte staat in beziehung auf landwirtschaft und nationalökonomie. Stuttgart. Traduction anglaise : The isolated state (1966). Pergamon Press, Oxford.

\section{Annexe}

\section{Les matrices de Poids}

Il existe plusieurs catégories de matrices de poids spatiales dont les principales sont les matrices de contiguité, les matrices de distance et les matrices des $k$ plus proches voisins.

Matrices de contiguïté. Deux unités spatiales sont contiguës si elles possèdent une frontière commune. On aura alors $w_{i j}=1$ si les unités spatiales sont contiguës à l'ordre 1 et $w_{i j}=0$ sinon. Une unité spatiale ne pouvant pas être contiguë avec ellemême, la diagonale de la matrice de poids $W$ ne contient que des valeurs nulles. De façon plus générale, deux unités spatiales $i$ et $j$ sont contiguës à l'ordre $k$ si $k$ est le nombre de frontières à traverser pour aller de $i$ à $j$. Parmi ces matrices de contiguïté, on distingue trois catégories. Le critère de différenciation est un critère de déplacement dont la terminologie a été empruntée au jeu d'échecs (Cliff \& Ord, 1981): le déplacement selon le critère de la tour consiste à considérer comme voisines deux unités spatiales représentées par des polygones partageant un même côté ; le déplacement selon le critère du fou consiste à considérer comme voisines deux unités spatiales représentées par des polygones partageant un même sommet; le déplacement selon le critère de la reine consiste à considérer comme voisines deux unités spatiales représentées par des polygones partageant un même sommet ou un même côté. Ce type de matrices nécessite donc qu'il n'y ait pas d'unité spatiale isolée au sein de l'espace étudié. 
Matrices de distance. Dans ces matrices, l'intensité des interactions entre deux unités spatiales est supposée dépendendante de la distance entre les centroïdes de ces unités spatiales. La matrice de poids $W$ est alors construite à partir d'une fonction décroissante de la distance entre les observations. Plusieurs indicateurs peuvent être utilisés selon la définition de la distance (distance à vol d'oiseau, indices d'accessibilité, etc.). Quant aux formes fonctionnelles, les plus utilisées sont la fonction exponentielle inverse, une fonction de l'inverse de la distance (Le Gallo, 2002) ou encore une combinaison d'une mesure de distance et de la longueur relative de la frontière commune entre deux unités spatiales pour tenir compte de l'irrégularité du zonage comme l'ont proposé Cliff \& Ord (1981). Ces matrices peuvent être utilisées en présence d'unités spatiales isolées.

Matrices des $k$ plus proches voisins. Egalement mobilisable en présence d'unités spatiales isolées au sein de l'espace étudié, les matrices des $k$ plus proches voisins sont basées sur une notion de distance également mais qui contrairement aux matrices de distance ne sont pas symétriques. On aura alors $w_{i j}=1$ si le centre de la zone $j$ est l'un des $k$ plus proches voisins de $i$ et $w_{i j}=0$ sinon. Le paramètre $k$ est fixé a priori. Cette méthode est notamment adaptée lorsque les tailles des unités spatiales sont très hétérogènes.

\section{Statistique de Moran}

La statistique / de Moran (Moran, 1948; 1950) s'écrit de la manière suivante :

$$
I=\frac{N}{S_{0}} \frac{\sum_{i} \sum_{j} w_{i j}\left(x_{i}-\bar{x}\right)\left(x_{j}-\bar{x}\right)}{\sum_{i}\left(x_{i}-\bar{x}\right)^{2}}
$$

avec: $\quad N$ le nombre total d'observations ;

$i$ l'unité de référence et $j$ l'unité voisine du point $i$;

$x_{i}$ l'observation dans l'unité spatiale $i$ et de moyenne $\frac{1}{N} \sum_{i} x_{i}$;

et

$$
S_{0}=\sum_{i} \sum_{j} w_{i j} \text { un facteur d'échelle avec } w_{i j} \text { un élément de la }
$$

matrice de poids $W$. Si $W$ est standardisée alors $S_{0}=N$. 
Tableau des statistiques I de Moran pour différentes matrices de poids

\begin{tabular}{lcccc}
\hline \multicolumn{4}{l}{ Tableau A. Statistiques I de Moran pour différentes matrices de poids } \\
\hline $\begin{array}{c}\text { Matrice de } \\
\text { poids }\end{array}$ & $I$ de Moran & $\begin{array}{c}\text { Espérance } \\
\text { de } I\end{array}$ & $\begin{array}{c}\text { Ecart-type } \\
\text { Valeur }\end{array}$ & $\begin{array}{c}\text { standardisée du I } \\
\text { de Moran }\end{array}$ \\
\hline k plus proches voisins & $E(I)$ & $\sigma(I)$ & $Z^{a}$ \\
2 voisins & 0,2885 & $-0,0024$ & 0,0460 & 6,2765 \\
3 voisins & 0,3155 & $-0,0024$ & 0,0370 & 8,5864 \\
4 voisins & 0,3102 & $-0,0024$ & 0,0319 & 9,8432 \\
5 voisins & 0,2758 & $-0,0024$ & 0,0287 & 9,6582 \\
6 voisins & 0,2664 & $-0,0024$ & 0,0270 & 9,9631 \\
7 voisins & 0,2705 & $-0,0024$ & 0,0255 & 10,6527 \\
8 voisins & $\mathbf{0 , 2 6 9 8}$ & $-\mathbf{0 , 0 0 2 4}$ & $\mathbf{0 , 0 2 2 4}$ & $\mathbf{1 2 , 2 2 4 2}$ \\
9 voisins & 0,2511 & $-0,0024$ & 0,0218 & 11,6826 \\
10 voisins & 0,2435 & $-0,0024$ & 0,0213 & 11,4633 \\
11 voisins & 0,2281 & $-0,0024$ & 0,0208 & 11,0971 \\
12 voisins & 0,2287 & $-0,0024$ & 0,0200 & 11,4889 \\
Distance euclidienne & & & 11,2295 \\
Distance & 0,1924 & $-0,0024$ & 0,0173 & \\
\hline
\end{tabular}

${ }^{a}$ La valeur standardisée de la statistique I de Moran $(Z)$ est donnée par : $Z=\frac{I-E(I)}{\sigma(I)}$

\section{Indicateurs locaux d'association spatiale (LISA)}

Selon Anselin (1995b), un indicateur local d'association spatiale est toute statistique satisfaisant les deux conditions suivantes :

- l'indicateur local d'association spatiale donne une indication sur le regroupement spatial significatif de valeurs similaires autour de chaque observation ;

- la somme des statistiques de LISA associées à toutes les observations est proportionnelle à un indicateur global d'association spatiale.

La combinaison de l'information obtenue par le diagramme de Moran et la significativité des LISA, permet alors d'obtenir des cartes de significativité montrant les unités spatiales associées à un LISA significatif. Chaque quadrant du diagramme de Moran est reporté sur ces cartes où les unités spatiales sont différenciées par un code couleur selon le quadrant concerné (Anselin \& Bao, 1997). On utilise alors une 
version locale de la statistique mesurant l'autocorrélation spatiale globale. La version locale du I de Moran est, pour chaque unité spatiale $i$ la suivante (Anselin, 1995b) :

$$
I_{i}=\frac{\left(x_{i}-\bar{x}\right)}{m_{0}} \sum_{j} w_{i j}\left(x_{j}-\bar{x}\right) \quad \text { avec } m_{0}=\frac{\sum_{i}\left(x_{i}-\bar{x}\right)^{2}}{N}
$$

La distribution pour ces statistiques ne pouvant être approximée par une loi normale, l'inférence statistique doit être basée sur l'approche des permutations conditionnelles (Anselin, 1995b), c'est-à-dire que la valeur de $x_{i}$ pour la localisation $i$ est maintenue fixée tandis que les valeurs restantes sont permutées sur toutes les autres unités spatiales. Les probabilités critiques ainsi obtenues pour les statistiques de Moran correspondent à des pseudo-niveaux de significativité.

\section{Calcul du consentement marginal à payer}

Le consentement marginal à payer (CMP) - ou prix implicite - se calcule à partir des coefficients estimés des variables concernées et du montant moyen de l'indemnité de substitution (Kim et al, 2003; Mueller \& Loomis, 2008). Lorsque la forme fonctionnelle est une double-log, le CMP associé aux variables continues est donné par:

$$
C M P_{x_{k}}=\hat{\beta}_{k} \times \frac{\bar{P}}{\bar{Z}}
$$

avec : $\quad k=1, \ldots, K, \hat{\beta}$ le coefficient estimé de la caractéristique étudiée, $\bar{P}$ l'indemnité moyenne et $\bar{Z}$ la moyenne des valeurs prises par la caractéristique étudiée.

Quant aux consentements marginaux à payer des variables binaires, ils se calculent de la façon suivante (Terra, 2005) :

$$
C M P_{x_{k}}=\left(e^{\hat{\beta}_{k}}-1\right) \times \bar{P}
$$

